\title{
MODEL-BASED METHOD FOR IMPROVING THE ACCURACY AND REPEATABILITY OF ESTIMATING VASCULAR BIFURCATIONS AND CROSSOVERS FROM RETINAL FUNDUS IMAGES
}

\author{
Chia-Ling Tsai ${ }^{l}$, Charles V. Stewart ${ }^{1}$, Howard L. Tanenbaum ${ }^{2}$, and Badrinath Roysam ${ }^{1}$ \\ ${ }^{1}$ Rensselaer Polytechnic Institute, Troy, New York 12180. \\ ${ }^{2}$ The Center for Sight, 349 Northern Blvd., Albany, NY 12204.
}

\begin{abstract}
A model-based algorithm, termed exclusion region and position refinement (ERPR), is presented for improving the accuracy and repeatability of estimating the locations where vascular structures branch and cross over, in the context of human retinal images. The goal is two fold. First, accurate morphometry of branching and crossover points (landmarks) in neuronal/vascular structure is important to several areas of biology and medicine. Second, these points are valuable as landmarks for image registration, so improved accuracy and repeatability in estimating their locations and signatures leads to more reliable image registration for applications such as change detection and mosaicing. The ERPR algorithm is shown to reduce the median location error from 2.04 pixels down to 1.1 pixels, while improving the median spread (a measure of repeatability) from 2.09 pixels down to 1.05 pixels. Errors in estimating vessel orientations were similarly reduced from 7.2 degrees down to 3.8 degrees. These improvements are especially significant for real-time image registration applications for which computationally expensive refinement approaches such as sum of squared difference (SSD) registration can be avoided.
\end{abstract}

Index Terms: Biomedical Image Analysis, Retinal Images, Landmarks, Bifurcations, Crossovers, Feature Extraction, Feature Stability, Feature Refinement, Image Registration, and Mosaic Synthesis.

\section{Correspondence:}

Prof. Badrinath Roysam,

Rm JEC 6046, ECSE Department,

Rensselaer Polytechnic Institute,

Troy, New York 12180-3590, USA.

Phone: 518-276-8067,

Fax: 518-276-6261,

Email: roysam@ecse.rpi.edu 


\section{Introduction}

The quantitative analysis of branched structures such as neurons and vasculature is important to several areas of biology and medicine [1,2,3,4,5,6,7,8,9]. Fig.1 shows an example from our prior work. Panel (a) is the image of a human retina as viewed by a common clinical instrument known as a fundus camera [10]. Panels ( $\mathrm{d} \& \mathrm{e})$ are the result of automatic tracing of the vasculature [5,9]. This group has also developed three-dimensional (3-D) extensions of these methods in the context of tracing neurons from confocal microscope images [11,6]. These examples are drawn from a much broader application base. For example, extracting blood vessel structures can be used to quantify the progress of angiogenesis in tumor growth [12]. Extracting neuronal structure can reveal the number of axons, dendrites, and spines, their lengths, and the amount and frequency of branching $[13,14,15,16]$.

Branching and crossover points in neuronal/vascular structures are of special interest from the standpoint of biology and medicine [17, 18, 19, 3, 20]. One such application is the early diagnosis of hypertension by measuring changes in select vascular branching and crossover regions [21, 22]. Another example is the study of early development of the retinal vasculature, and its evolution under various pathologies and applied conditions [23, 24, 25, 26, 27].

Branching and crossover points are also important from a purely image analysis standpoint. The locations of these points, if known to be stable, are valuable as features (i.e., landmarks) for image registration and mosaicing. In retinal imaging, these points are known to be stable unless the retina is detached. The often-unique pattern of angles of intersection can be used as landmark signatures [20, 28, 29, 30, 31, 32]. Registered images can be used to reveal retinal changes and pathologies [33, 28]. Mosaics of retinal images provide high-resolution, wide extent imaging of the retina for diagnosis of pathologies of the retinal periphery [29, 30, 34]. High-speed image registration can provide the basis for computer-aided instrumentation for ophthalmic surgery [20, 35, 36]. Fig. 2 illustrates instances of registration and mosaicing. Panels (a) and (b) show two views of the same retina. Panel (c) is the result of registering the images in panels (a) and (b) using a landmarks-based image registration algorithm. In this work, the branch points of the vasculature, and also the crossover points, are used as the basis for registration. Panel (d) shows a complete retinal montage (mosaic) constructed by registering a series of retinal images to form a wide-angle high-resolution image of the retina [30]. Such montages not only have direct 
clinical usefulness [34], but also are useful for spatial mapping and referencing, for controlling ophthalmic instruments, for procedures such as laser retinal surgery [10].

Crucial to the performance of image registration algorithms is the accuracy and repeatability with which vascular crossing and branching locations (landmarks) can be extracted. The landmarks when placed in correspondence constrain the image-to-image transformation that must be estimated to register the images. Of particular interest, in retinal image registration, at least six landmark correspondences are needed to completely constrain a 12-parameter transformation model that accounts for the curvature of the retinal surface [29]. In these algorithms, more landmark correspondences are needed to increase the probability of correct registration, and also registration accuracy. When a limited number of landmarks are available, the accuracy of landmark locations is crucial. Complementarily, accurate estimation of landmark locations and branching angles can enable registration using fewer, but more reliable landmarks. This can result in greatly accelerated registration. Our interest is in achieving highly accurate registration at frame rates $[37,20,38]$.

In prior work, we have described an extremely fast and robust class of algorithms for tracing the retinal vasculature in an exploratory manner [5,9]. These algorithms are based on an underlying model of the vasculature in which a fragment of the vasculature is characterized by two relatively straight, anti-parallel edges with either an intensity peak or an intensity valley in between.

The present paper extends our prior work by greatly improving the accuracy and repeatability with which branching and crossover points are extracted. It is based on the observation that while the "anti-parallel edges" model is an excellent basis for tracing the linear portions of the vasculature, it is not an adequate basis for describing the branching and crossing points. Indeed, these are locations where the model breaks down, introducing errors in the estimated landmark coordinates, and consequently the angles as well. This phenomenon is illustrated in Fig. 3. The column on the left shows greatly magnified views of selected vascular branch locations. The image is overlaid with annotations generated by our earlier tracing algorithm. In the vicinity of the branch point, the vessels are not well modeled as bound by a pair of approximately parallel boundaries with opposite gradient values. The dotted lines indicate the traces generated by the algorithm. The column on the right shows the improved results generated by the ERPR algorithm described in the present work. 
We address the problem of accurate landmark extraction by introducing a new model for the geometric structure of landmarks and by developing an algorithm that uses this model to accurately and robustly estimate landmark location (see Fig. 4). In this model, a landmark is defined as a location, a size, and a set of directions. The location is the point closest to the centerlines of all blood vessels that meet to form the intersection. The size is the width of the intersection region. The directions are the orientations of the blood vessels relative to the landmark location. The location, size and direction parameters of this model are estimated iteratively starting from the initial meeting of traces; unreliable centerline points falling within the intersection region are excluded from this estimate. The model and resulting algorithm produce substantially more accurate, repeatable landmarks than our initial method. Examples are shown in Figs. 3 \& 7.

The organization of the paper is as follows. Section II summarizes the exploratory tracing algorithm [5] on which the landmark extraction mechanism is built. Section III summarizes the limitations of prior methods. Section IV describes the details of the new landmark model and the estimation techniques. Section V quantifies the improved repeatability of the landmark locations using the new methods, and demonstrates its impact on our retinal image registration method $[29,30]$. Section VI summarizes the work.

\section{Background to the Present Study - Exploratory Tracing Algorithms}

Two main approaches are described in the literature for extracting vascular landmarks in angiograms. The first approach requires extensive pixel processing, and relies on adaptive segmentation, followed by skeletonization and branch point analysis, or the use of interest operators [e.g., 20], often using specialized hardware [39]. The second approach exemplified by this paper, is called vectorization, or exploratory tracing (see Fig. 1). This method relies on a recursive tracing of the vasculature based on a localized model. This approach has been shown to be much faster (e.g. video frame rates are readily achieved), more adaptive, and more practical for implementation on conventional and parallel MIMD computers [40]. It also requires the fewest parameter settings, scales well with image size, and provides useful partial results [9]. 
The exploratory tracing algorithm, and detailed in [5], proceeds in three stages.

Step 1 (seed point initialization): The algorithm analyzes the image along a coarse grid (see Fig. 1b) to gather gray-scale statistics (contrast and brightness levels) and to detect seed locations on blood vessels -- gray-scale minima between opposite signed 1-D edges. False seed points are filtered out by testing for the existence of a pair of sufficiently strong parallel edges with opposite gradient values. For this, a set of directional kernels [5] is applied to the seed's neighboring points along the grid line, and the two strongest responses are found. The initial point is filtered out if the two strongest responses do not both exceed a sensitivity threshold, or if the directions of the two strongest edges are not sufficiently similar (within $\pm 22.5^{\circ}$ ). On average, about $40 \%$ of the initial points are filtered out by this procedure.

Step2 (recursive tracing): The second stage, illustrated in Fig. 1(c) is a sequence of recursive tracing steps that are initiated at each of the filtered seed points, and proceed along vessel centerlines using an update equation of the following form:

$$
p^{k+1}=p^{k}+\alpha\left[\cos \left(\frac{2 \pi s^{k}}{N}\right), \quad \sin \left(\frac{2 \pi s^{k}}{N}\right)\right]^{T}+\beta^{k},
$$

where $k$ is the iteration count, $p^{k}$ and $p^{k+1}$ denote the current and new $(x, y)$ locations of the trace, $\alpha$ is a step size, $s^{k} \in\{0,1,2 \ldots N-1\}$, is an integer index specifying one of $N$ discretized angular directions (usually $N=16$ ), and $\beta^{k}$ is a lateral displacement vector that centers the new point $p^{k+1}$ on the vessel. In Fig. 1(c), this is illustrated for a pair of intersecting vessels. The left and right directional kernels at $0^{\circ}$ and $45^{\circ}$ are also illustrated for the lower branch in Fig. 1(c). In equation (1), $s^{k}$ is estimated as the angle at which the correlation kernels produce the highest response. These maximum responses are computed by performing a local search along a line perpendicular to the current trace.

Step 3 (landmark extraction): The tracing that starts from a seed point continues until the end of the vessel is reached or until the centerline of the tracing intersects a previously detected vessel centerline. Landmarks are placed at intersections of traces and at locations where three or more centerline traces (see Fig. 1(d)) meet. In the former case, the location is the actual intersection; in the latter the location is the centroid of the trace endpoints. These landmarks are 
characterized by this location and by the orientations of the traces meeting to form the landmark. Example traces and landmarks are shown in Fig. 1(d \& e).

The reader is referred to [5] for further details, including methods to minimize computation, a description of algorithms for pooling the traced segments, avoiding repeated searches, detecting branch points, crossovers, and end points, and correction of traces near branch points. Also described in this reference are methods for automatic estimation of the grid size used for initial exploration, the threshold used to terminate tracing, and the step size $\alpha$.

\section{Limitations of Prior Methods}

The design of the landmark extraction technique in the exploratory tracing algorithm summarized above is conceptually simple, and effective in terms of detection ability. However, it suffers from two limitations relating to the accuracy and repeatability of estimating the intersection coordinates and angles.

These limitations arise primarily from the fact that the anti-parallel edge model on which the tracing algorithm is based, is no longer valid very close to branching and crossover points due to the rounded nature of the junctions (see Fig. 4). Consequently, when the recursive tracing steps (equation (1)) approach a junction, the estimation of the centerline of the vessels is less accurate. In other words, trace points become unstable very close to intersections. The peak responses of the left and right templates often occur for many different orientations as they begin to overlap intensity values from two or more different blood vessels. This may result in uncertain, even apparently random placement of centerline points (Figs. $3 \&$ 7) occasionally.

This estimation is also influenced by variations in the locations of the seed points, implying that the repeatability with which the centerline is estimated is impaired as well. Finally, errors in estimating the point of intersection have a pronounced effect on the accuracy with which the intersection angles are estimated. The issues related to landmark accuracy are illustrated in Fig. 3. The issues related to repeatability are illustrated in Fig. 7. 


\section{Exclusion Region and Position Refinement (ERPR) Method}

\subsection{A Model for Intersections}

As noted above, the failure of the anti-parallel edges model near intersections is the primary source of error. The solution proposed here is to introduce an explicit model of the structure of a landmark and an estimation technique that estimates the parameters of this model. The goals of this paper are to develop such a model and estimation technique, include them in the current exploratory tracing algorithm, and demonstrating the effectiveness of the resulting algorithm.

The proposed landmark model, illustrated in Fig. 4, consists of three parts:

1. A circular exclusion region: This region models the region of intersection of the blood vessels. In this region, the anti-parallel model of the blood vessels is violated. Therefore, traces computed in this region are not used.

2. The landmark location: This is defined as the $(x, y)$ point nearest the extrapolation of the centerlines of the vessels that meet to form the landmark.

3. Orientation vectors: The set of blood vessel orientations that meet to form the intersection. These orientations are defined relative to the landmark location.

The exclusion region radius is estimated once, but the other parameters -- the landmark location and the blood vessel orientations -- are estimated iteratively. The following sub-sections provide a more detailed description of these items, the estimation algorithms, and the relevant notation.

\subsection{Algorithm Overview}

The starting point for the estimation process is the endpoint of a trace when either it intersects the boundary of another blood vessel or it meets at least two other trace endpoints. From this endpoint, the algorithm gathers information about neighboring traces, estimates the initial landmark point, determines the exclusion radius, and estimates the initial blood vessel orientations. This initializes an iterative process that alternates steps of re-estimating traces and orientations of blood vessels outside the exclusion region, and then re-estimating the landmark point from the blood vessels. Together these steps ensure that the traces outside the intersection 
are more accurate, that multiple trace points are used in estimation, and that ultimately the final landmark location is estimated more accurately.

\subsection{Gathering Information About Neighboring Traces}

The estimation technique for a single landmark starts from a trace endpoint and a set of neighboring traces. Fig. 4 illustrates the terminology and notation used here.

A trace is defined as a sequence of centerline points detected during recursive tracing starting from a single seed point. Let $T$ be the set of all traces (across the entire image), $t \in T$ be a single trace, and $P(t)=\left[p_{t, 0}, p_{t, 1}, \ldots p_{t, i} \ldots.\right]$ be the sequence of centerline points on trace $t$. The centerline points on trace $t$ are denoted $p_{t, i}$. The ending point of the trace is denoted $p_{t, e}$. The set of neighboring traces, denoted by $N\left(p_{t, e}\right)$, is the set of traces having at least one other centerline point close to endpoint $p_{t, e}$. Finally, let $w_{t, i}=w\left(p_{t, i}\right)$ be the width of the blood vessel at each centerline point, computed easily during recursive tracing.

Detecting a trace endpoint $p_{t, e}$ and triggering the landmark extraction technique is done using a slight modification to the original stopping criteria. Tracing of a vessel terminates if it encounters boundaries of other existing traces (Fig. 5a). The advantage of this is that at a branch region of a thick vessel, the trace from one branch can possibly miss another trace and run parallel to it, resulting in an intersection far away from the true intersection region. By stopping the second trace at the bound of the first, we avoid this problem. Tracing also terminates if the template response is too weak. This can occur either when the blood vessel is no longer visible or, sometimes, when tracing has intersected a different blood vessel.

Following termination of tracing for a specific trace $t *$ and endpoint $p_{t^{*}, e}$ the set of neighbor traces, denoted $N\left(p_{t^{*}, e}\right)$ is found. This set is defined as

$$
N\left(p_{t^{*}, e}\right)=\left\{t \in T \mid \min _{p \in P(t)}\left\|p_{t^{*}, e}-p\right\| \leq w(p)\right\} .
$$

In other words, the set $N\left(p_{t^{*}, e}\right)$ contains all traces having at least one centerline point, $p$, closer to $p_{t^{*}, e}$ than the blood vessel width at $p$. If $N\left(p_{t^{*}, e}\right)$ contains only trace $t^{*}$, we relax the condition by extracting traces falling into the region centered at $p_{t, e}$ with a default radius. In that case, $N\left(p_{t^{*}, e}\right)$ is redefined as 


$$
N\left(p_{t^{*}, e}\right)=\left\{t \in T \mid \min _{p \in P(t)}\left\|p_{t^{*}, e}-p\right\| \leq c\right\} \quad,
$$

where $c$ is a constant and set empirically to 10 pixels in our implementation, based on the observation that the average width of the vessels in our images is less than 10. The motivation for equation 3 is to compensate for the early termination of tracing due to failure of the antiparallel edge model. The search for neighbor traces is expedited by storing trace points in regularly spaced, $n \times n$ bins covering the entire image.

The last step in the gathering process is to compute the set of closest points to $p_{t^{*}, e}$ from the traces in $N\left(p_{t^{*}, e}\right)$. Indeed, this determines if a landmark should even exist. Determine this set as:

$$
C\left(p_{t^{*}, e}\right)=\left\{p \mid \min _{p \in P(t)}\left\|p_{t^{*}, e}-p\right\|, t \in N\left(p_{t^{*}, e}\right)\right\} .
$$

\subsection{Initializing the Landmark Model Parameters}

During the initialization process, the landmark location, the exclusion region radius, and the blood vessel orientations are estimated in turn. The exclusion region radius remains fixed throughout the computation.

Initializing the landmark location depends on the contents of the set of closest points $C\left(p_{t^{*}, e}\right)$ :

If $C\left(p_{t^{*}, e}\right)$ consists of at most one other endpoint besides $p_{t^{*}, e}$, then no landmark is placed here. (Subsequent tracing from a different seed point could eventually place a third endpoint here, leading to the establishment of a landmark.)

If $C\left(p_{t^{*}, e}\right)$ contains only endpoints (at least three) then the initial landmark location, denoted by $q^{0}$, is the centroid of the endpoints.

If $C\left(p_{t^{*}, e}\right)$ contains a point $p$ that is not an endpoint, then trace $t *$ stopped when it reached the middle of another trace. In this case, the initial landmark location $q^{0}$ is taken as this closest point $p$. Furthermore, the trace $t \in N\left(p_{t^{*}, e}\right)$ containing $p$ is split into two traces, and both stored in $N\left(p_{t^{*}, e}\right)$. Please see Fig. 5 for an illustration.

Next, the exclusion region radius, $r *$, is estimated. Intuitively, the exclusion region should have a diameter at least as wide as the width of the thickest vessel in the intersection. Since the width of a vessel varies, and is less reliable as the vessel approaches the intersection, we define the width of a trace, $w(t)$, as the median value of widths of all trace points on $t$. The exclusion 
radius $r *$ is then defined as the maximum of the trace widths for all traces in $N\left(p_{t^{*}, e}\right)$. There is no need to refine $r^{*}$ because it does not depend significantly on the landmark location or other landmark parameters.

The final step in initialization is estimating the blood vessel orientations near the intersection. This orientation is denoted $\theta(t)$ for each trace $t \in N\left(p_{t^{*}, e}\right)$. The initial value of this orientation, $\theta^{0}(t)$, is found by fitting a line to points on trace $t$ that are just outside the exclusion region. This is described in more detail below because it is exactly the same computation as used in the iterative procedure.

\subsection{Iterative Estimation of Model Parameters}

The trace centerlines, blood vessel orientations, and landmark location are estimated iteratively. In the $j^{\text {th }}$ iteration, the refined trace centerline locations and blood vessel orientations are $P^{j}(t)$ and $\theta^{k}(t)$ for $t \in N\left(p_{t^{*}, e}\right)$. Note that $P^{0}(t)$ is the initial sequence of trace points. The landmark location in iteration $j$ is $q^{j}$.

The first step in each iteration, i.e., for $j \geq 1$, is to re-estimate the trace centerline points near landmark location $q^{j-1}$, but outside the exclusion region. This procedure is called back-trace refinement, and is illustrated in Fig. 5. For each trace $t \in N\left(p_{t^{*}, e}\right)$, a seed point on the boundary of the exclusion region is found, and then the recursive tracing procedure described in Section II is run for a small number of steps (e.g., $n=5$ ) away from the intersection. The seed point for each back trace is given by:

$$
q^{j-1}+\left[r^{*} \cos \theta^{j-1}(t), r^{*} \sin \theta^{j-1}(t)\right]
$$

which is where a ray from the previous landmark location in the previous blood vessel direction intersects the boundary of the exclusion region. The refined trace points replace the corresponding trace points to form the set $P^{j}(t)$ from $P^{j-1}(t)$ at the $j^{\text {th }}$ iteration.

The next step is to re-estimate the orientation of the blood vessel associated with each trace $t$ from the trace points and landmark location. The $n$ points in $P^{j}(t)$ closest to $q^{j-1}$ but outside the exclusion region are found. Except when back-trace refinement is ignored, as discussed below, 
these points are exactly the new trace points just computed. For each trace, these points are placed in a set $S$ together with the previous landmark location, $q^{j-1}$, and the orthogonal leastsquares regression line $L^{j}(t)$ is computed from this set. The previous landmark location is added to ensure stability, especially for intersections with acute angles. The new orientation is taken from the line parameters.

The final step is to estimate the new landmark location, $q^{j}$. This is computed from the line parameters $L^{j}(t)$ that have been just estimated. The specifics of doing this depend on the line representation, but are equivalent. For example, if $L^{j}(t)$ is represented by a unit normal $\hat{\eta}^{j}(t)$, and point $p^{k}(t)$ (any point on the line will do), then $q^{k}$ is the point minimizing the following least-squares criterion

$$
\sum_{t \in N\left(p_{t^{*}, e}\right)}\left[\hat{\eta}^{k}(t) \cdot\left(p^{k}(t)-q^{k}\right)\right]^{2}
$$

This point is unique unless all lines are parallel, which of course cannot happen in this application.

Unfortunately, on rare occasions, back-trace refinement produces a dramatically different set of traces from the original set. This occurs because of low image contrast or nearby untraced vessels, or a combination of these. An example is shown in Figure 6. We detect this situation through its affect on the estimated point $q^{j}$. If the distance between successive points, $q^{j}$ and $q^{j-1}$, is greater than half the exclusion region radius, $r^{*}$, then back-trace refinement is ignored, the set of trace points reverts to the set from the initial iteration (i.e. $P^{j}(t)=P^{0}(t)$ ), and the remainder of the $j^{\text {th }}$ iteration is repeated.

The iterative process terminates when the landmark location stabilizes -- specifically, $\left\|q^{j}-q^{j-1}\right\|<0.25$ pixels -- or the number of iterations exceeds a limit.

\subsection{Simplified Estimation of Landmark Model Parameters}

The most expensive (and unstable) part of the estimation process is back-trace refinement. It makes sense to consider the possibility of a simpler algorithm where back-trace refinement is 
removed from the iterative estimation process and only applied at the end. This simplified process proceeds as follows: The lines $L^{j}(t)$ are estimated for each trace $t \in N\left(p_{t^{*}, e}\right)$. Then, the refined landmark location $q^{j}$ is estimated from these lines. After the iterative process, a single full iteration is applied, including back-trace refinement, line estimation, and landmark location estimation.

Using the same test criterion as above, if the new location is too far from the previous one, the landmark location is restored to its previous value. Intuitively, this should work because it should be possible to obtain a reasonably accurate estimate of the landmark location from the traces outside the exclusion region, and allowing final accuracy of the centerline positions, trace orientations, and then landmark position to be achieved in just a single full iteration.

\section{Experimental Evaluation}

In this section, the accuracy and repeatability of the proposed ERPR technique is evaluated in the context of retinal image registration. These tests are all based on datasets from 18 different eyes, with 10-20 images in each data set. Each image is of size $1024 \times 1024$ pixels, and were captured using a Topcon IMAGENET digital camera system at the Center for Sight (Albany, New York).

The method for measuring the accuracy and repeatability of landmark estimation is based on a highly accurate image registration algorithm developed in prior work $[29,30,31,32]$ is used as a testbed and standard for measuring landmark estimation errors. This is a hierarchical registration technique that uses correspondences between landmarks in two different retinal images to estimate a 12-parameter, quadratic transformation mapping one retinal image onto another (for an example, see Fig. 2). The average alignment error of this registration algorithm, as measured by the distance between trace centerlines is 0.83 pixels on $1024 \times 1024$ images. This algorithm models the retina as a curved quadratic surface that is imaged by a weak-perspective uncalibrated camera. Our publications [29, 30, 31, 32, 41] provide a detailed error analysis for this algorithm, including a break down of error sources (modeling errors, tracing \& registration errors, and errors from descretization). 
The very last step of the registration algorithm noted above is especially significant. It is designed to correct the registration for errors in estimating landmark locations (based on the original tracing and landmark localization technique [5]). As the transformation estimate nears convergence, the grayscale intensities of regions surrounding landmark locations are matched using a localized sum-of-squared-differences (SSD) technique [42]. Even though this is nearly the last step, it is by far the most expensive, occupying near $90 \%$ of the total computation time, due to the pixel processing involved. The registration accuracy using the original landmarks is 1.59 pixels, and using the SSD matching technique is 0.83 pixels. Indeed, these observations served as a powerful motivation to explore methods to improve the estimation of landmark locations - the work reported in this paper. If the new landmarks are sufficiently accurate, then perhaps the expensive SSD matching can be eliminated.

The ability to register retinal images with sub-pixel accuracy in spite of errors in estimating the landmark locations (albeit at a high computational price) leads to several methods of evaluating the proposed Exclusion Region and Position Refinement (ERPR) algorithm.

- Repeatability Measurement: For a landmark that appears in two or more images, registering the images places the two different estimated positions in the same coordinate system. This gives us a measure of the repeatability of the landmark position, modulo the transformation error itself.

- Accuracy Measurement: We can compare the SSD refined positions obtained just prior to convergence to the estimated landmark positions as a further measure of position accuracy.

- We can analyze the effect of the accuracy of landmark positions on the estimate of the quadratic transformation.

- We can generate a qualitative, visual indication of the effectiveness of the ERPR technique by transforming many different images of the same landmark into the same coordinate system. Examples of this are shown in Fig. 7.

The first quantitative measure is the error between the same landmark estimated in registered pairs of images. Let $q_{1}$ be the landmark location in image $I_{1}$ and $q_{2}$ be the landmark location in image $I_{2}$. Let $\Theta_{1,2}$ be the estimated registration function mapping $I_{1}$ onto $I_{2}$, so that $\Theta_{1,2}\left(q_{1}\right)$ is the mapping of the landmark location into image $I_{2}$. The local distance $\left\|\Theta_{1,2}\left(q_{1}\right)-q_{2}\right\|$ gives one 
measure of the landmark error. The maximum difference in trace orientations between the mapping of the landmark at $q_{1}$ and the landmark at $q_{2}$ gives a second measure. By combining these measures over all landmarks in all registered image pairs, we obtain summary statistics on the repeatability of the estimated landmark parameters.

Table 1 shows these summary statistics for the original landmark detection technique and for several versions of our new ERPR technique, including the full method, the simplified method using just one iteration of back trace refinement, and a version where back trace refinement is not used at all. Several conclusions are immediately apparent. First, the ERPR method is twice as repeatable as the original method, both in terms of position and orientation. In fact, it may be higher because of the inherent error in the transformation. Second, there is little difference between the fully iterative ERPR and the simplified version where back trace refinement is used only once. Third, with no use of back-trace refinement the results are substantially worse. Based on the latter two conclusions, the remaining experiments will focus exclusively on the ERPR with one step of back trace refinement.

The second quantitative comparison is between the SSD-refined landmark positions that emerge at the end of registration and the positions estimated by either the original landmark technique, or by ERPR. Using corresponding point locations $q_{1}$ and $q_{2}$, as above, the SSD step during estimation of $\Theta_{1,2}$ refines the position of $q_{2}$ to match the transformation of a small region surrounding $q_{1}$. Call the new position $q_{2}^{\prime}$. The error measure is then $\left\|q_{2}-q_{2}^{\prime}\right\|$. Interestingly, this measure is not sensitive to minor errors in the transformation, and therefore an even better measure of the repeatability of the landmark position estimate. The disadvantage is that the SSD refinement gives no improvement in orientation accuracy.

The results of this measure taken over all correctly registered pairs from our data set are shown in Table 2. Both median and average errors are given. The advantage of the new ERPR method with just a single iteration of back-trace refinement is striking. The average error is 2.5 times lower and the median error is 1.9 times lower.

The final quantitative analysis is to consider the effect of the new landmark model and estimation technique on the registration algorithm itself. The major question is whether or not the landmark positions are accuracy enough to eliminate the SSD-refinement step altogether. SSDrefinement step in fact consists of two steps: one is the landmark position refinement as 
discussed above, and the second is SSD-based matching of landmarks detected in one image but missing in the other. The second step occurs after the first. Here is a summary of the registration accuracies:

1. Using the original landmark model and localization technique and no position refinement, the registration error is 1.59 pixels;

2. Using the original landmark model, and the first step of SSD refinement, the registration error is 0.97 ;

3. Using the original landmark model and both SSD refinement steps, the registration error is 0.83 pixels;

4. Using the new landmark model, ERPR, with a single iteration of back trace refinement at each landmark and no SSD refinement, the error drops to 1.21. This is certainly a dramatic improvement but not as accurate as the SSD steps. We conclude that when substantial registration accuracy is required, the SSD refinement is still the best (albeit computationally expensive) alternative. The proposed ERPR method is a reasonable tradeoff for applications that require speed of computation (e.g., [37]);

\section{Discussion and Conclusions}

We have presented a new model and algorithm for estimating the location and orientation of blood vessel landmarks -- branching and crossover points -- in retinal fundus images. This technique works in the context of our earlier algorithm for recursively tracing the blood vessel structure using an anti-parallel edge model. This earlier model does not accurately describe blood vessels near landmarks, which leads to instability in the estimated landmark positions. Our new model describes landmarks in terms of an intersection location, a circular intersection (exclusion) region over which the anti-parallel model does not apply, and a set of blood vessel orientations emerging from the landmark. The parameters of this model are initialized from locations where recursive traces meet or intersect, and then re-estimated iteratively, including the positions of traces near the intersection. Experiments in the context of retinal image registration showed the improved repeatability of landmark locations between different retinal images. They also showed that a single iteration of trace refinement was sufficient for estimation accuracy.

There exists several line junction and corner detection techniques in the literature of stereo vision and motion detection. Techniques include template matching [43, 44], gray-level dissimilarity measure [45], steerable filters [46], estimation of local line curvature [47, 48, 49], 
parametric model fitting [44] and parameterized feature detection by projecting local brightness distribution into the subspace [50]. Those techniques work either on gray-scale intensity by defining a junction as the point where two or more homogeneous surface patches are located within the neighborhood, or on curvature of the contour by extracting the junction as the point of local maximum change of gradient direction [51] estimates the density of nerve intersections by digitizing lines as row segments and deduce intersections from the adjacent overlapping segments. Our technique differs from others by extracting the junction as the closest point to the intersecting edges.

The new landmark model and estimation technique are useful in several ways. Intrinsically, there is value in improved repeatability and hence reliability of landmark position estimates. In practice, we showed that the new landmark positions improved registration accuracy substantially, but not as much as an expensive SSD-based position and matching refinement algorithm. Recently, however, we have begun to use the new landmark model and estimation technique in a slightly different context: invariant indexing for registration between images taken during retinal treatment procedures and a pre-computed mosaic of images taken during diagnosis [37]. Invariants -- measures on a point set that don't change when the image viewpoint changes -are computed from pairs or triples of landmarks. Here, the improved repeatability of landmark locations has proven extremely important. We anticipate similar benefits in other future applications.

Since only a small set of trace points along each trace is used for line approximation, variation in the location of any of the trace points can introduce considerable change in the direction of the line. As shown in Fig. 8, the curving of the vessels near the branching region results in a set of trace points with very different locations on different images. This in turn degrades the stability of the landmark positions. Further work is required to reduce the effect of curved vessels on the landmark approximation. One approach may be to fit higher-order curves (e.g. constrained quadratics) instead of lines.

\section{Acknowledgments}

The authors would like to thank the staff at the Center for Sight, especially photographers Gary Howe and Mark Fish, for extensive with image acquisition. We are thankful to Prof. Ali Can at the University of Inonu, Turkey for discussions and valuable suggestions. Various 
portions of this research was supported by the National Science Foundation Experimental Partnerships grant EIA-0000417, the Center for Subsurface Sensing and Imaging Systems, under the Engineering Research Centers Program of the National Science Foundation (Award Number EEC-9986821), the National Institutes for Health grant RR14038, and by Rensselaer Polytechnic Institute. 


\section{References}

[1] Coatrieux, J. L., Garreau, M., Collorec, R., and Roux, C., "Computer Vision Approaches for the Three-Dimensional Reconstruction: Review and Prospects," Critical Rev. Biomed. Eng., 22(1):1-38, 1994.

[2] Capowski, J. J., ed., “Computer Techniques in Neuroanatomy”, Plenum Press, New York, 1989.

[3] Robin, G. D., Paik, D. S., Johnston, P. C., and Napel, S., "Measurements of the Aorta and Its Branches with Helical CT, Radiology, Vol. 206, No. 3, pp. 823-829, 1999.

[4] Wilson, D. L., and Noble, J. A., "An Adaptive Segmentation Algorithm for Extracting Arteries and Aneurysms from Time-of-flight MRA data," IEEE Transactions on Medical Imaging, Vol. 18, No. 10, pp. 938-945, Oct 1999.

[5] Can, A., H. Shen, J. N. Turner, H. L. Tanenbaum, and B. Roysam, "Rapid Automated Tracing And Feature Extraction From Live High-Resolution Retinal Fundus Images Using Direct Exploratory Algorithms," IEEE Transactions on Information Technology in Biomedicine, vol. 3, no. 2, pp. 125-138, June 1999.

[6] Al-Kofahi, K., Lasek, S., Szarowski D., Pace, C., Nagy, G., Turner, J. N., Roysam, B., "Rapid Automated Three-dimensional Tracing of Neurons from Confocal Image Stacks," IEEE Transactions on Information Technology in Biomedicine, (accepted, to appear) 2001.

[7] Cohen, A. R., Roysam, B., and Turner, J. N., "Automated Tracing and Volume Measurements of Neurons from 3-D Confocal Fluorescence Microscopy Data," Journal of Microscopy, Vol. 173, Pt. 2, February 1994.

[8] Wink, O., Niessen, W. J., Viergever, M. A., "Fast Delineation of Vessels in 3-D Angiographic Images," IEEE Transactions on Medical Imaging, vol. 19, No. 4, pp. 337-346, 2000.

[9] Shen, H., Roysam, B., Stewart, C. V., Turner, J. N., and Tanenbaum, H. L., "Optimal Scheduling of Tracing Computations for Real-time Vascular Landmark Extraction from Retinal Fundus Images," IEEE Transactions on Information Technology in Biomedicine, vol.5, No. 1, March 2001. 
[10] Federman, J. L., editor. Retina and Vitreous. The C.V. Mosby Company, St. Louis, 1988.

[11] Pawley, J. (ed.), Handbook of Confocal Microscopy, Plenum Press, New York, 1995.

[12] Saaristo, A., Karpanen, T., and Alitalo, K., "Mechanisms Of Angiogenesis And Their Use In The Inhibition Of Tumor Growth And Metastasis," Oncogene, 19(53):6122-9, Dec 2000.

[13] E. Korkotian and M. Segal, "Structure-Function Relations In Dendritic Spines: Is Size Important?" Hippocampus, 10(5):587-95, Oct 2000.

[14] A. McAllister, A., "Cellular And Molecular Mechanisms Of Dendrite Growth," Cereb Cortex, 10(10):963-7, Oct 2000.

[15] Glaser, J. R., and Glaser, E., "Neuron Imaging with Neurolucida - A PC-Based System for Image Combining Microscopy", Computerized Medical Imaging and Graphics 14:307-317, 1990.

[16] Turner, J. N., Shain, W., Szarowski, D. H., Lasek, S., Sipple, B., Pace, C., Al-Kofahi, K., Can, A. and Roysam, B., "Confocal Light Microscopy of Brain Cells and Tissue: Image Analysis \& Quantitation," Acta Histochemica et Cytochemica, vol. 32, No. 1, pp. 5-11, 1999.

[17] Kyriacos, S., Nekka, F., and Cartilier, L., "Insights into the Formation Process of the Retinal Vasculature", Fractals, Vol. 5, No. 4, pp. 615-624, 1997.

[18] Sequeira, J., Ebel, R., and Schmitt, F., "3-D Modeling of Tree-like Anatomical Structures," Computerized Medical Imaging and Graphics, vol. 17, no. 4/5, pp. 333-337, 1993.

[19] Schwartz, R. B., Jones, K. M., Chernoff, D. M., Mukherji, S. K., Khorasani, R., Tice, H. M., Kikinis, R., Hooton, S. M., Steig, P. E., and Polak, J. F., “ Common Carotid Artery Bifurcation: Evaluation with Spiral CT,” Radiology, Vol. 185, No. 2, pp. 513-519, 1992.

[20] Becker, D. E., Can, A., Tanenbaum, H. L., Turner, J. N., and Roysam, B. "Image Processing Algorithms For Retinal Montage Synthesis, Mapping, And Real-Time Location Determination,” IEEE Transactions on Biomedical Engineering, 45(1), 1998.

[21] Tso, M. O. M, and Jampol, L. M. "Pathophysiology Of Hypertensive Retinopathy," Ophthalmologica 89:1132, 1982.

[22] Parida, L., Geiger, D., and Hummel. R., "Junctions: Detection, Classification, And Reconstruction," IEEE Transactions on Pattern Analysis and Machine Intelligence, 20(7): 687698, July 1998. 
[23] Family, F., Masters, B., and Platt, D., "Fractal Pattern Formation In Human Retinal Vessels," Physica D, Vol. 38, 1989, 98-103.

[24] Toeledo, R., Radeva, P., C Von Land, and Villanueva, J., "3D Dynamic Model of the Coronary Tree," IEEE Computers in Cardiology, 1998, Vol.25, p.777.

[25] Neumann, F., Schreiner, W., and Neumann, M., "Computer Simulation Of Coronary Arterial Trees," Advances in Engineering Software, Vol.28, 1997, pp.353-357.

[26] Onuki, T., and Nitta, S., "Computer Simulation of Geometry and Hemodynamics of Canine Pulmonary Arteries," Annals of Biomedical Engineering, Vol.21, pp. 107-115, 1993.

[27] Sherman, T., “On Connecting Large Vessels to Small,” Journal of Gen. Physiology, Vol. 78, Oct. 1981, p.431.

[28] Berger J. W., Patel T. R., Shin D. S., Piltz J. R., Stone R. A., "Computerized Stereo Chronoscopy and Alternation Flicker to Detect Optic Nerve Head Contour Change," Ophthalmology. $2000 \mathrm{Jul}$; 107(7): 1316-20.

[29] Can, A., Stewart, C. V., Roysam, B., and Tanenbaum, H. L., "A Feature-Based Robust Hierarchical Algorithm for Registration of Pairs of Images of the Curved Human Retina," (accepted, to appear) IEEE Transactions on Pattern Analysis and Machine Intelligence, 2001.

[30] Can, A., Stewart, C. V., Roysam, B., and Tanenbaum, H. L., "A Feature-Based Algorithm for Joint, Linear Estimation of High-Order Image-to-Mosaic Transformations: Mosaicing the Curved Human Retina," (accepted, to appear) IEEE Transactions on Pattern Analysis and Machine Intelligence, 2001.

[31] Can, A., C. V. Stewart, B. Roysam, "Robust Hierarchical Algorithm for Constructing a Mosaic from Images of the Curved Human Retina," Proceedings IEEE Computer Society Conference on Computer Vision and Pattern Recognition, Fort Collins, Colorado, June 1999.

[32] Can, A., C. V. Stewart, B. Roysam, H. L. Tanenbaum, "A feature-Based Technique for Joint, Linear Estimation of High-Order Image-to-Mosaic Transformations: Application to Mosaicing the Curved Human Retina," Proceedings IEEE Computer Society Conference on Computer Vision and Pattern Recognition, Hilton Head Island, South Carolina, June 2000 
[33] Goldbaum, M. H., Kouznetsova, V., Coté, B. L., Hart, W. E., and Nelson, M., “Automated Registration Of Digital Ocular Fundus Images For Comparison Of Lesions," in SPIE: Ophthalmic Technologies III, vol. 1877, 94-99, 1993.

[34] Mahurkar, A. A., Vivino, M. A., Trus, B. L., Kuehl, E. M., Datiles, M. B., and KaiserKupfer. M. I., “Constructing Retinal Fundus Photomontages," Investigative Ophthalmology and Visual Science, 37(8):1675-1683, July 1996.

[35] Barrett, S. F., Wright C. H. G., Jerath, M. R., “Computer-aided Retinal Photocoagulation System," Journal of Biomedical Optics, vol. 1, pp.83-91, 1996.

[36] Berger, J. W., and Shin D. S., “Computer Vision Enabled Augmented Reality Fundus Bio-microscopy," Ophthalmology, vol. 106, no. 10, Oct. 1999.

[37] Shen, H., Lin, G., Stewart, C. V., Tanenbaum, H. L., and Roysam, B., "Frame-Rate Spatial Referencing Based on Invariant Indexing and Alignment with Application to Laser Retinal Surgery," Proceedings of the IEEE Computer Society Conference on Computer Vision and Pattern Recognition, Kauai, Hawaii, December 11-13, 2001.

[38] Shen, H., Indexing Based Frame-Rate Spatial Referencing Algorithms : Application To Laser Retinal Surgery, Ph.D. Thesis, Rensselaer Polytechnic Institute, Troy, NY 12180, Dec 2000.

[39] Polli, R., and Valli, G., "An Algorithm For Real-Time Vessel Enhancement And Detection," Computer Methods and Programs in Biomedicine, vol. 52, pp. 1-22, 1997.

[40] Balduf, E., Real-Time Algorithms for a Laser Retinal Surgery System - Implementation on the Silicon Graphics Octane Dual-Processor System, M.S. Thesis, Rensselaer Polytechnic Institute, 1998.

[41] Can, A., "Robust Computer Vision Algorithms For Registering Images From Curved Human Retina," Ph.D. thesis, Rensselaer Polytechnic Institute, Troy, New York 12180, Aug 2000.

[42] Brown, L.G., "A Survey of Image Registration Techniques," Surveys(24), No. 4, December 1992, pp. 325-376.

[43] Bigun, J., “A Structure Feature For Some Image Processing Application Based On Spiral Functions," CVGIP, 51:166-194, 1990. 
[44] Rohr, K., "Recognizing Corners By Fitting Parametric Models," International Journal of Computer Vision, 9(3):213-230, 1992.

[45] Cooper, J., Venkatesh, S., and Kitchen, L., "Early jump-out corner detectors," IEEE Transactions on Pattern Analysis and Machine Intelligence, 15(8):823-829, Aug 1993.

[46] Simoncelli, E., and Farid, H., "Steerable Wedge Filters For Local Orientation Analysis," IEEE Transactions on Image Processing, 5(9): 1377-1383, 1996.

[47] Francois, D., and Djemel, Z., "Extracting Line Junctions From Curvilinear Structures,” In Proceedings of Geoscience and Remote Sensing Symposium, IGARSS, IEEE 200 international, volume 4, pages 1672-1674, 2000.

[48] Kitchen, L., and Rosenfeld, A., "Gray Level Corner Detection," Pattern Recognition Letters, (1): 95-102, Dec 1982.

[49] Mokhtarian, F., and Suomela, R., "Robust Image Corner Detection Through Curvature Scale Space," IEEE Transactions on Pattern Analysis and Machine Intelligence, 20(12):13761382, Dec 1998.

[50] Nayar, S., Baker, S., and Murase, H., "Parametric Feature Detection," Proceedings IEEE Conference on Computer Vision and Pattern Recognition, pages 471-478, 1996.

[51] Sebok, T., Roemer, L., and G. Malindzak, Jr., “An Algorithm For Line Intersection Identification," Pattern Recognition, 13(2):159-166, 1981. 


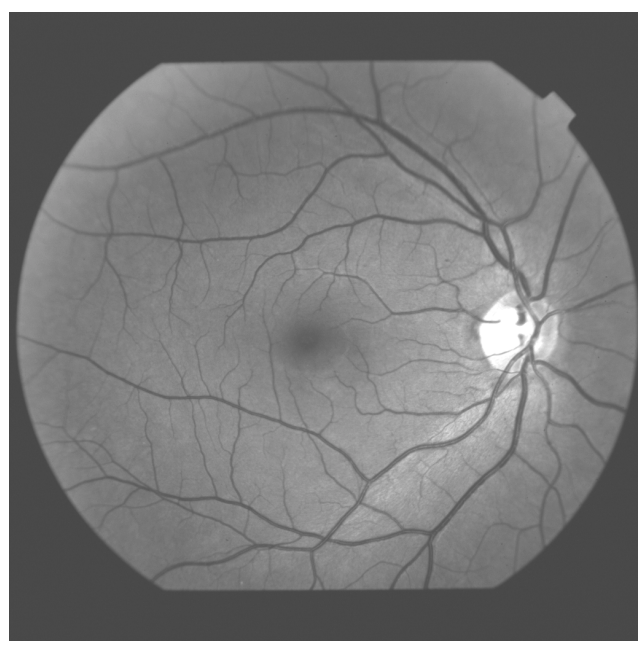

(a)

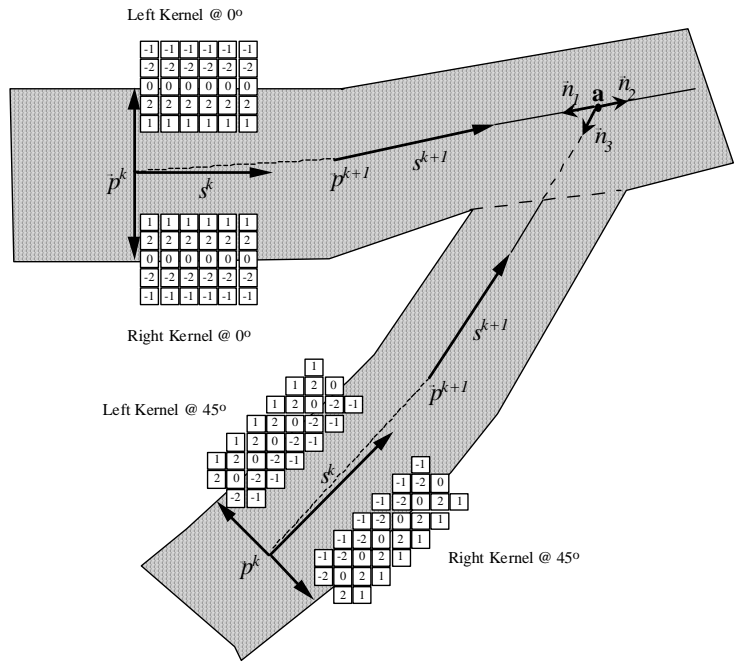

(c)

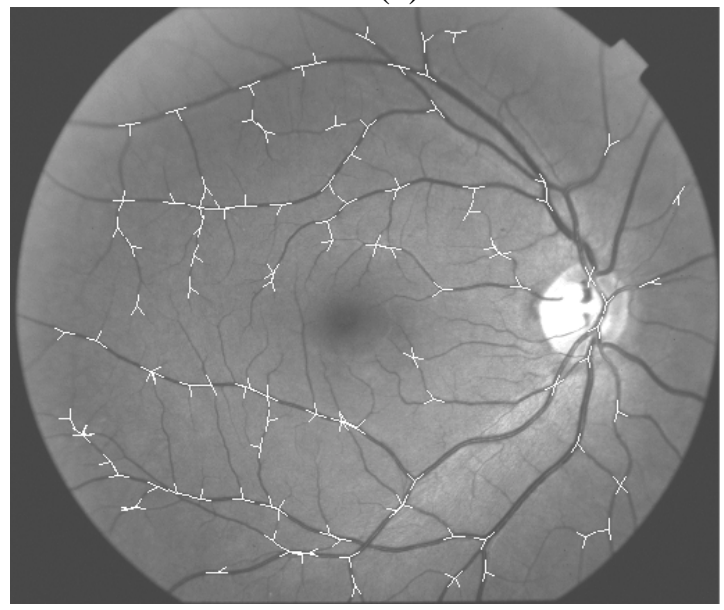

(e)

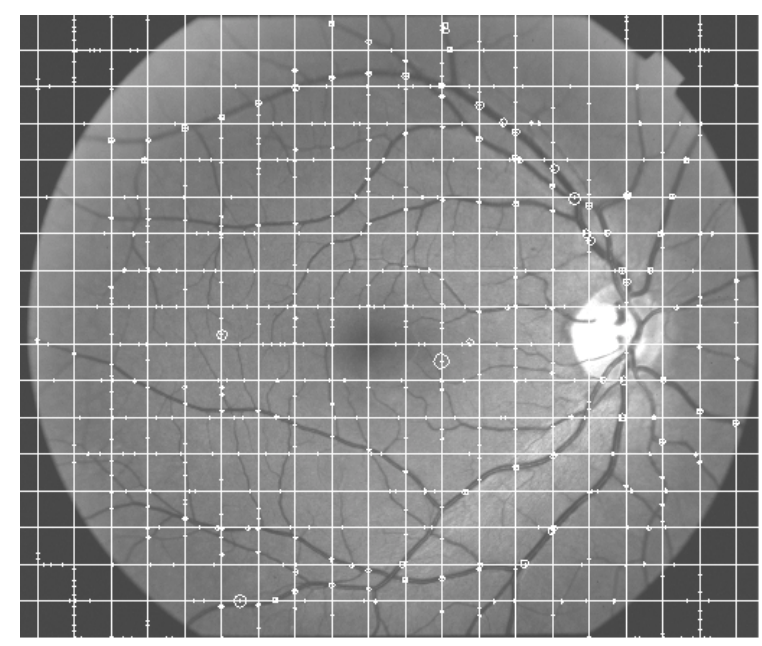

(b)

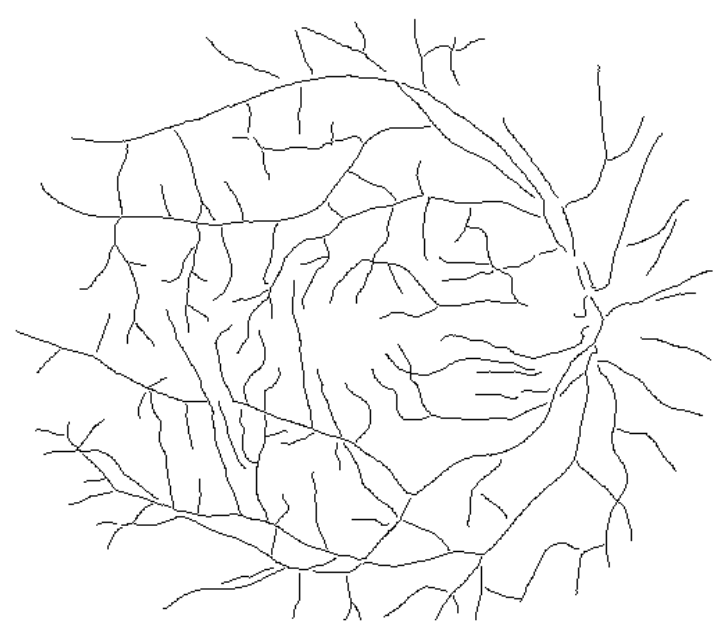

(d)

Figure 1: Example from prior work, illustrating fast tracing of branched structures. (a) Image of a fundus image, overlaid with results from the initial step of the automatic tracing algorithm in which the image is sampled along a sparse grid to estimate image contrast, and verified intersections with vessels (seeds), shown as circled dots; (b) Illustrating the recursive tracing algorithm based on a model that expects a pair of anti-parallel edges; (c) Result of automatic tracing, shown as vessel centerlines; (d) Detected bifurcations and crossover points. These are of prime interest as landmarks (features) for image registration (illustrated in Figure 2).

Tsai et al., 2001 


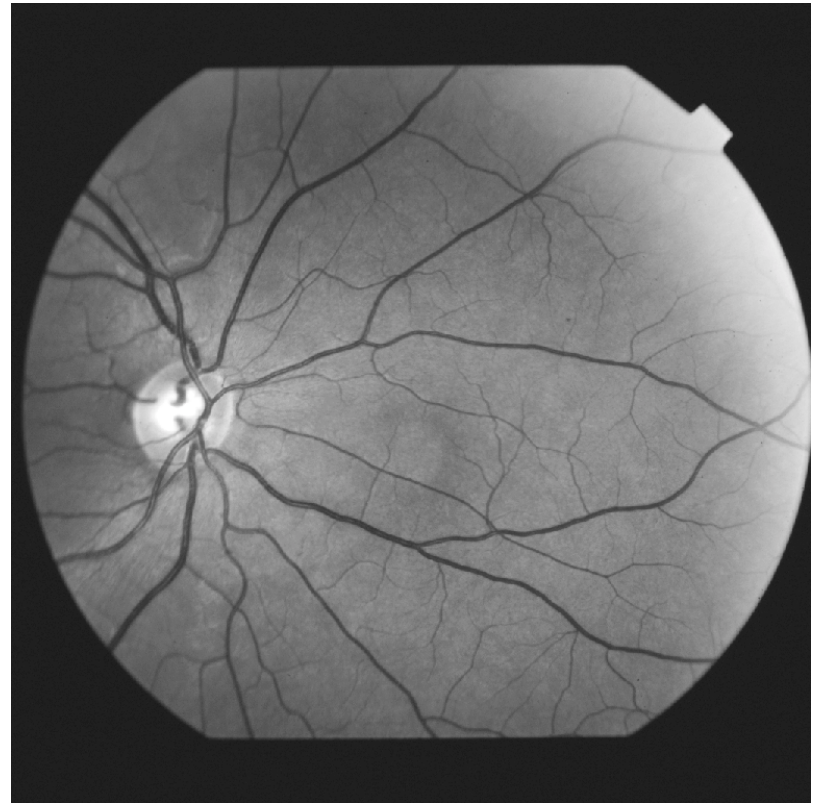

(a)

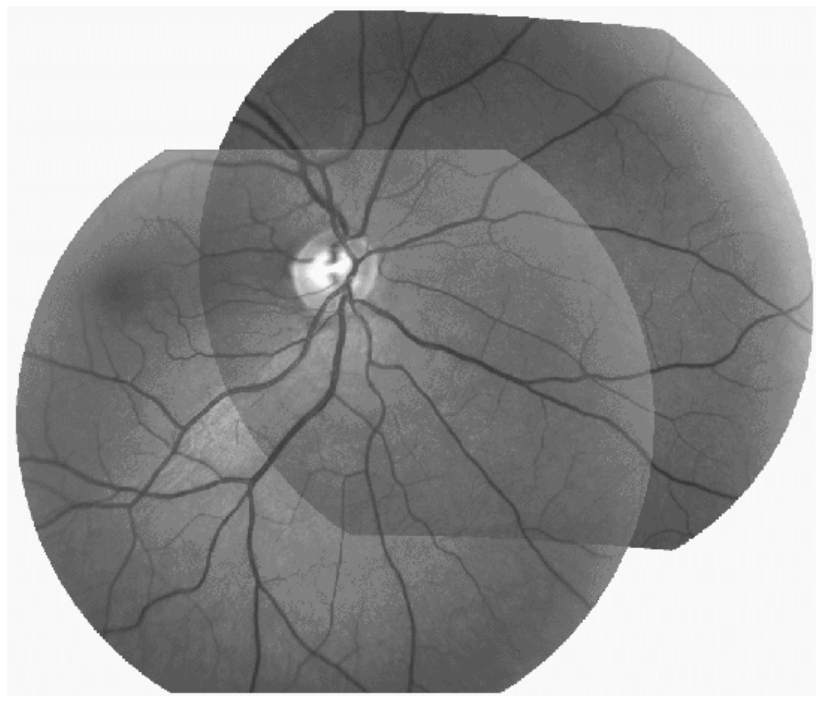

(c)

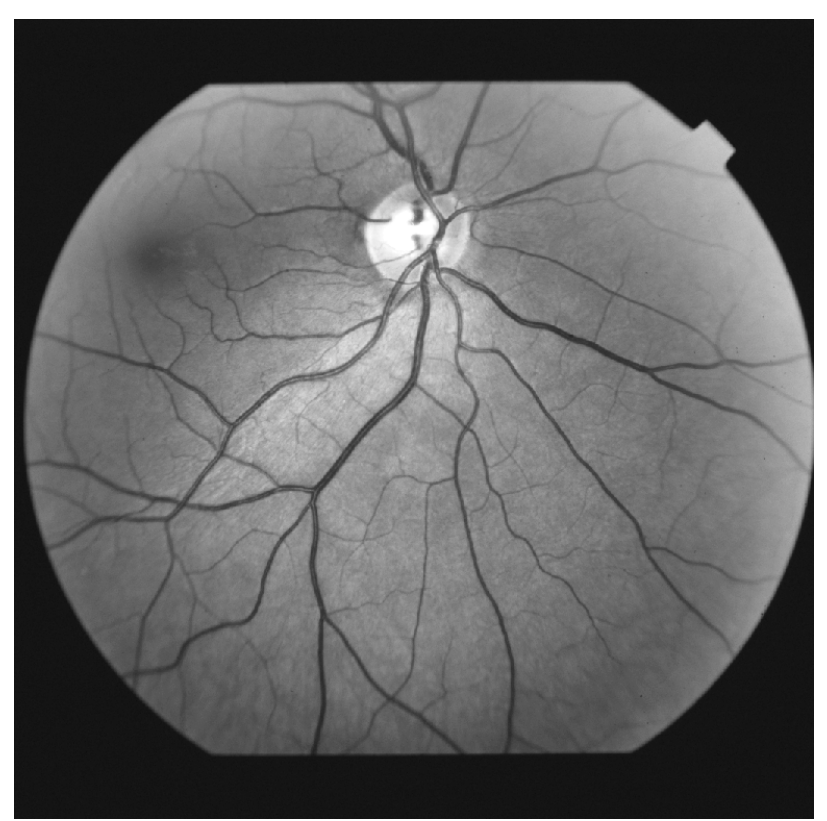

(b)

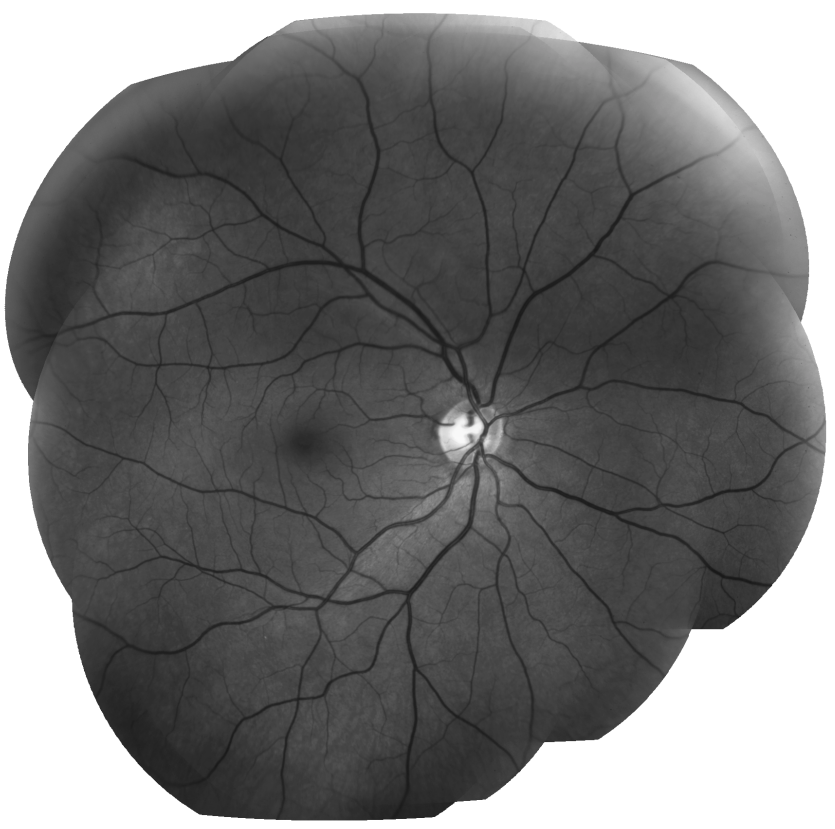

(d)

Figure 2: Illustrating retinal image registration and mosaicing. Panels (a) and (b) are fundus images taken at the same time with approx. 50\% overlap (c) the results of registering panels (a) and (b), displayed as a mosaic. Panel (d) is a complete mosaic of the same retina. Accurate registration to sub-pixel accuracy requires precise and repeatable estimation of image features (landmarks), and their signatures (intersection angles and thickness values). 

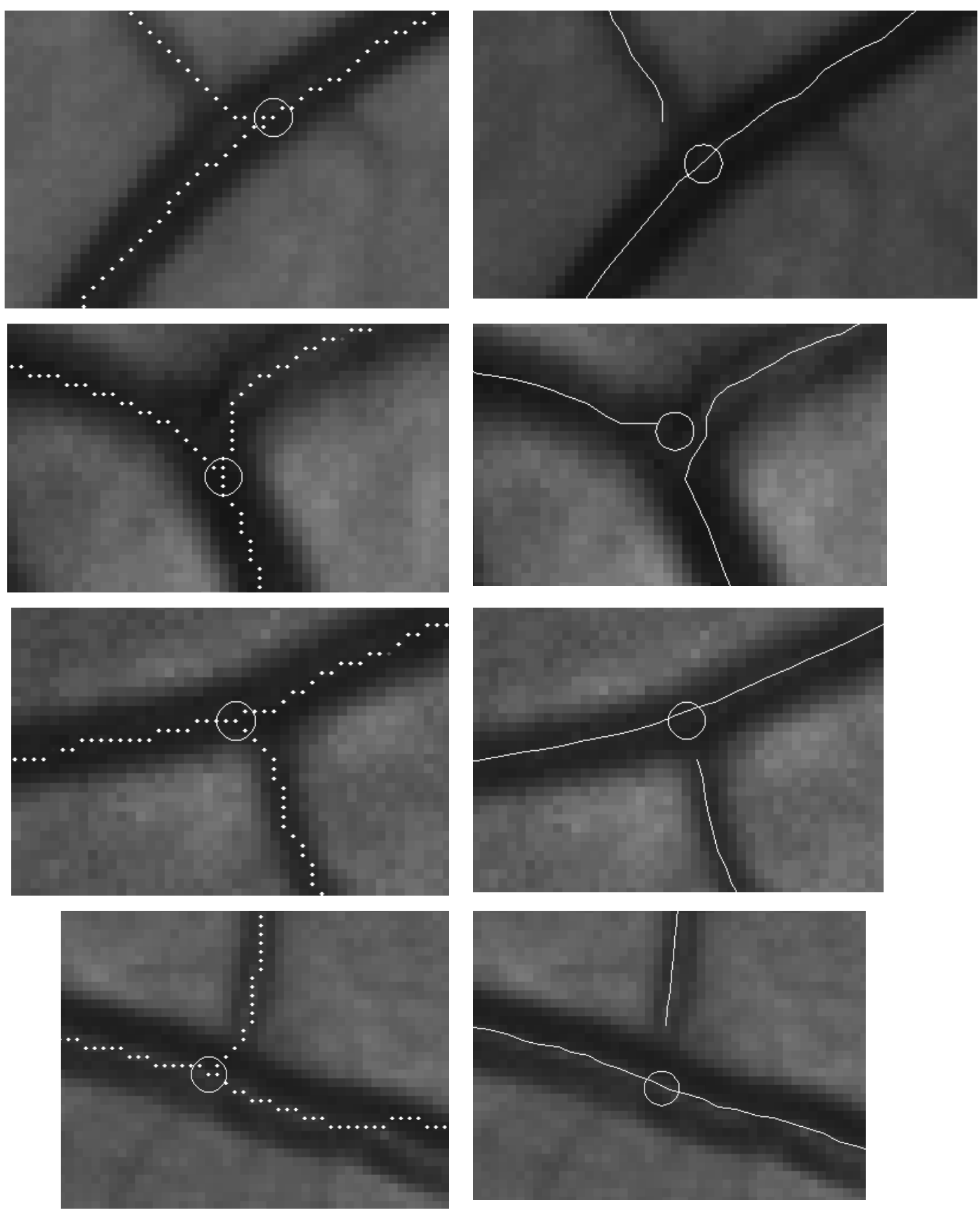

Figure 3: Illustrating the issue of landmark location accuracy. The left column shows enlarged close-up views of three landmarks, overlaid with the results of tracing from our earlier algorithm. As the tracing steps approach the intersection, the anti-parallel edges model that holds well for the straight portions of the vasculature fails, leading to errors in estimating the intersection position and angle. The column on the right shows results produced by the enhanced algorithm (ERPR) presented in this paper. This algorithm estimates the locations of the intersections, and the angular signatures more accurately using a model (illustrated in Figure 4). The detected intersection is the center of the overlaid circle. 


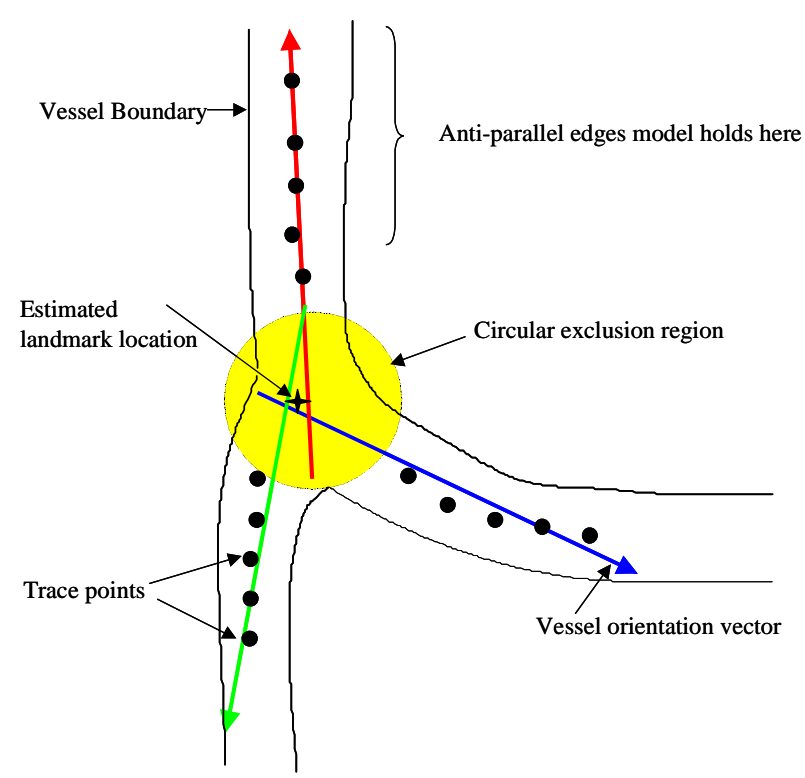

(a)

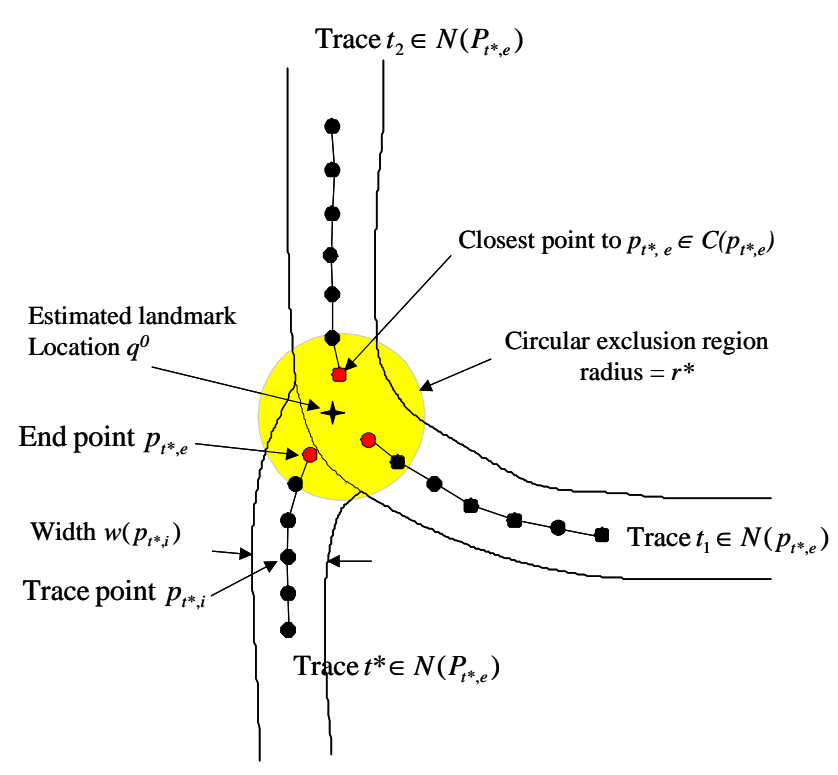

(b)

Figure 4: Illustrating the landmark (intersection) model. Panel (a) shows the terminology. The vessels are assumed to exhibit pairs of anti-parallel edges/boundaries far away from intersections. The circular exclusion region is the region over which the anti-parallel edges model for vessels is not valid. It is also the region where the proposed model is valid and useful. Traces farther away from the exclusion region provide estimates of the local vessel orientations. The estimated landmark location is indicated by a star symbol. Panel (b) shows the mathematical notation. 


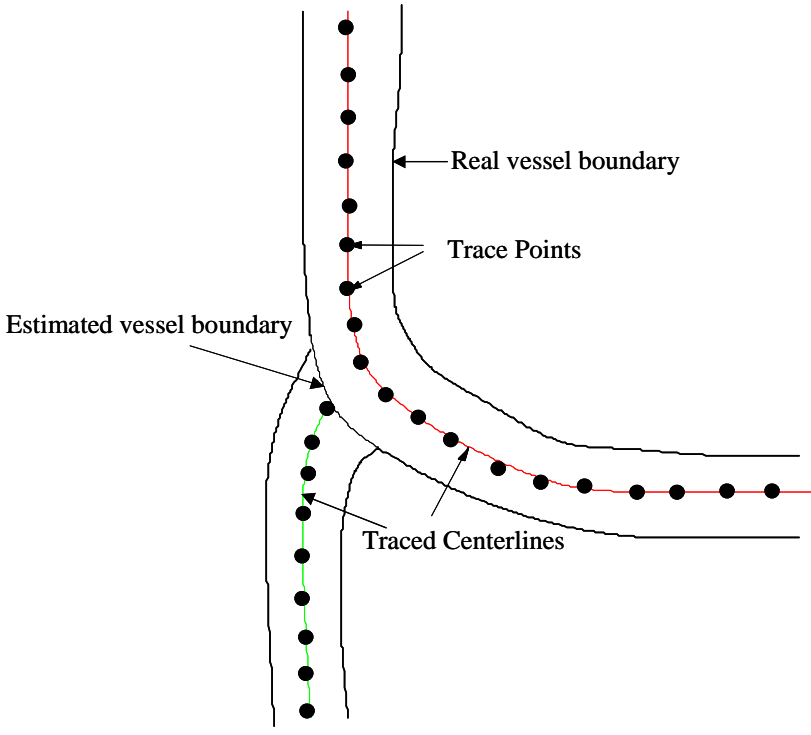

(a)

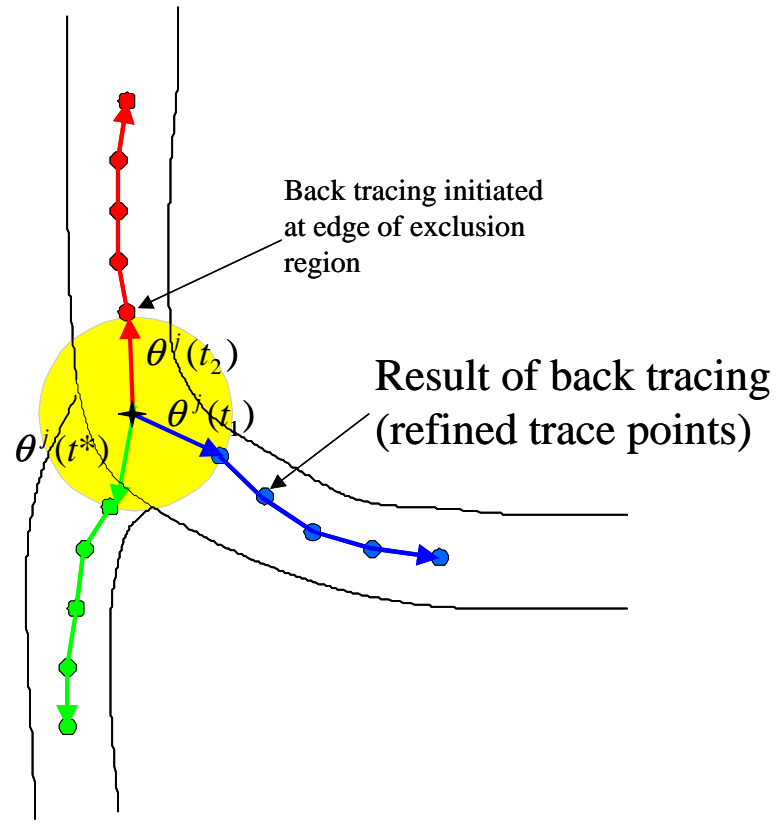

(c)

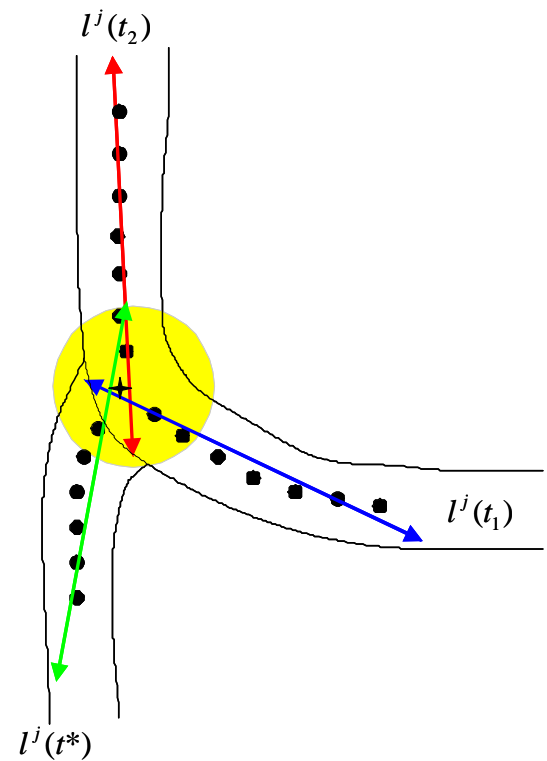

(b)

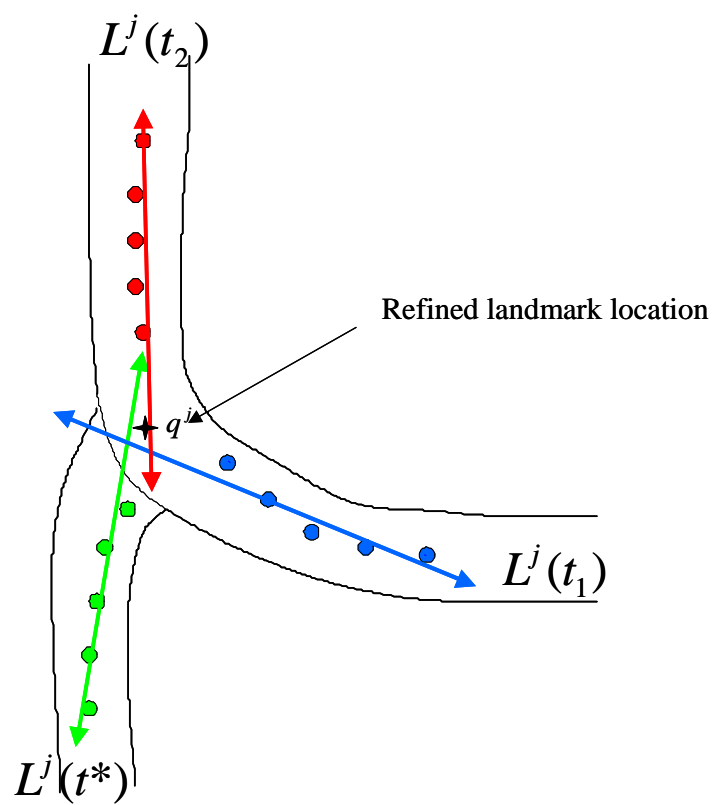

(d)

Figure 5: Illustrating the steps in estimating model parameters: (a) The result of initial tracing; (b) Showing the exclusion region in yellow, and method for initializing the back tracing. Lines, denoted $l^{j}\left(t_{1}\right), l^{j}\left(t_{2}\right)$, and $l^{j}\left(t^{*}\right)$ are fit to the previously traced centerline points; (c) showing new traces from back-tracing, initiated from points that are estimated based on the angles of the fitted lines, and just outside the exclusion region; (d) The refined landmark location is estimated by fitting lines denoted $L^{j}\left(t_{1}\right), L^{j}\left(t_{2}\right)$, and $L^{j}\left(t^{*}\right)$, and finding the point $q^{j}$ that is closest to these lines.

Tsai et al., 2001 


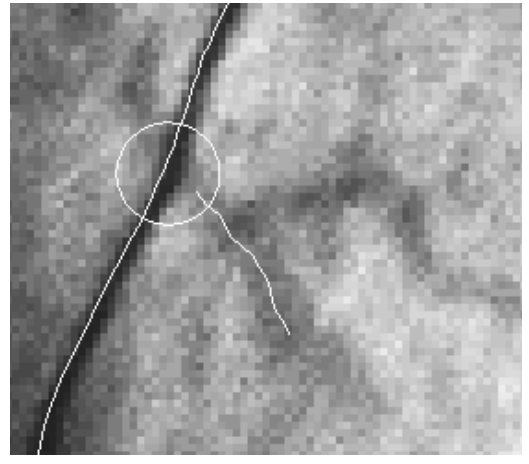

(a)

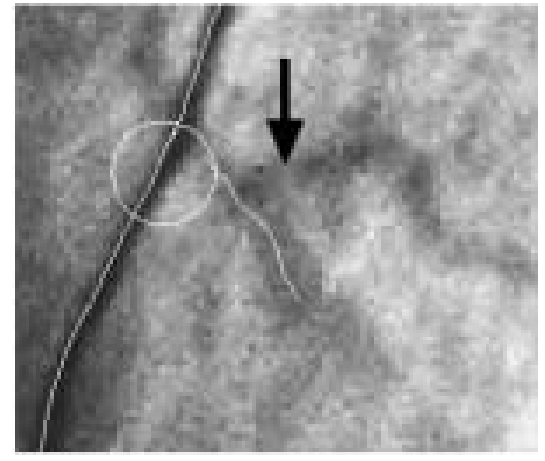

(b)

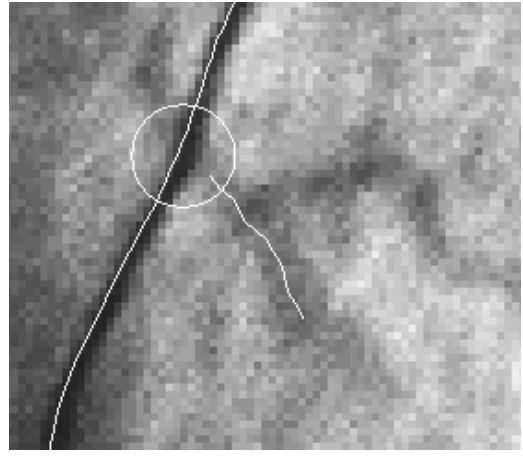

(c)

Figure 6: Illustrating the effect of nearby traces on back-tracing procedure. The circle shows the exclusion region. (a) Result from the previous iteration. (b) The arrow shows the vessel that misleads back tracing and results in a drastic shift in the location approximation. (c) The result without back tracing. If the distance between estimated location from successive iterations, $q^{j}$ and $q^{j-1}$, is greater than half the exclusion region radius, $r^{*}$, then back-trace refinement is ignored, the set of trace points reverts to the original trace sequence. 
Table 1: Landmark repeatability experiment. The original landmark method is compared to three versions of the new ERPR method: the full iterative refinement; the simplified version with one step of back trace refinement; and an even simpler version with no back trace refinement whatsoever.

\begin{tabular}{|c|c|c|c|c|}
\hline & \multicolumn{2}{|c|}{ Location Distance } & \multicolumn{2}{|c|}{ Max Orientation Difference } \\
\hline & $\begin{array}{l}\text { Median Error } \\
\text { (Pixels ) }\end{array}$ & $\begin{array}{l}\text { Mean Error } \\
\text { (Pixels) }\end{array}$ & $\begin{array}{l}\text { Median Error } \\
\text { (Degrees) }\end{array}$ & $\begin{array}{l}\text { Mean Error } \\
\text { (Degrees) }\end{array}$ \\
\hline Original & 2.09 & 2.21 & 7.20 & 8.63 \\
\hline ERPR, full & 1.05 & 1.34 & 3.75 & 4.80 \\
\hline ERPR, simplified & 1.07 & 1.33 & 4.33 & 5.43 \\
\hline ERPR, no back-trace & 1.28 & 1.51 & 5.03 & 6.04 \\
\hline
\end{tabular}

Table 2: Landmark position vs. SSD refined position: comparing the original landmark estimation technique to the new ERPR method with a just one step of back trace refinement.

\begin{tabular}{||c||c|c|}
\hline \multicolumn{1}{||c|}{} & \multicolumn{2}{|c|}{ Location Error } \\
\cline { 2 - 3 } & $\begin{array}{r}\text { Median } \\
\text { (Pixels) }\end{array}$ & $\begin{array}{l}\text { Mean } \\
\text { (Pixels) })\end{array}$ \\
\hline \hline Original & 2.04 & 1.76 \\
\hline \hline ERPR, simplified & 1.10 & 0.72 \\
\hline \hline
\end{tabular}



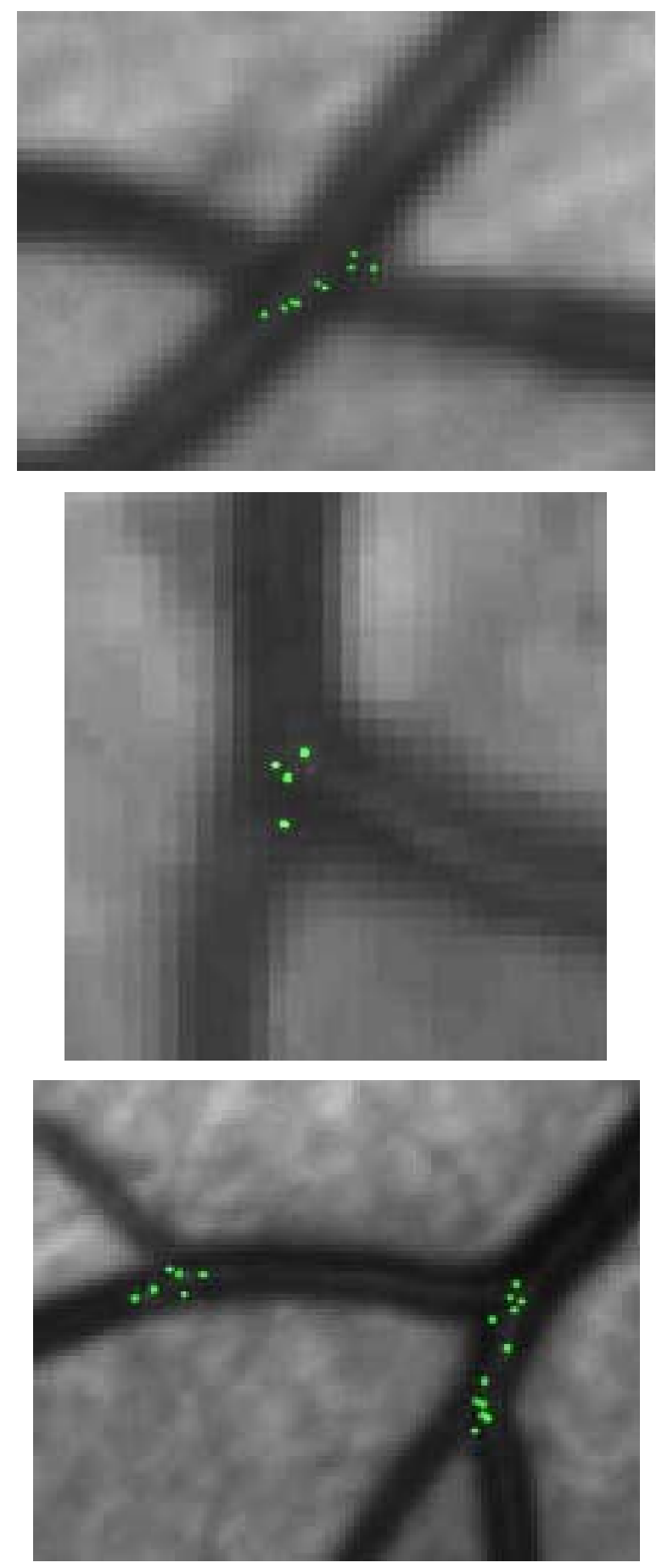
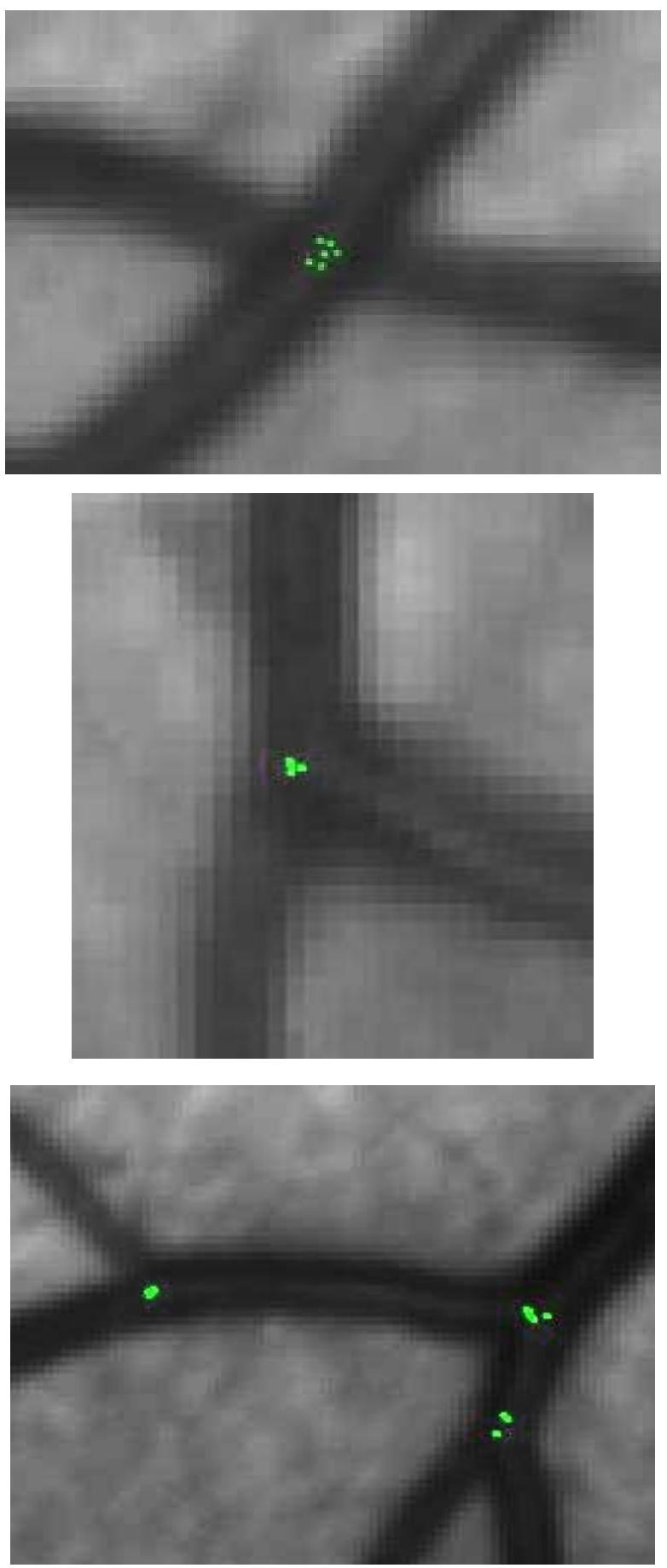

Figure 7: The green dots on the vessels are the landmarks mapped from the fundus images. The left column shows three examples from the original method. The right column shows the corresponding results from the ERPR algorithm for the same image regions. Observe the substantial improvement in the repeatability with which the locations are estimated. This is important for image registration applications, especially with real-time implementations. 


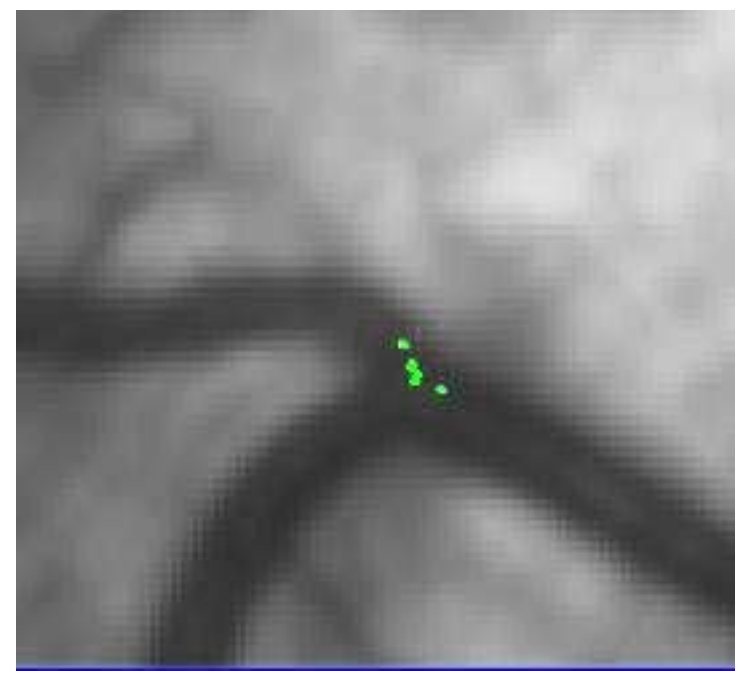

Figure 8: Example showing failure of the ERPR algorithm. A high curvature of one or more vessels in the immediate vicinity of the intersection region leads to reduced repeatability of landmark estimation. 